Article

\title{
On a New Class of Laplace-Type Integrals Involving Generalized Hypergeometric Functions
}

\author{
Wolfram Koepf ${ }^{1}$, Insuk Kim ${ }^{2, *}$ and Arjun K. Rathie ${ }^{3}$ \\ 1 Department of Mathematics, University of Kassel, Heinrich-Plett-Str. 40, D-34132 Kassel, Germany \\ 2 Department of Mathematics Education, Wonkwang University, Iksan 570-749, Korea \\ 3 Department of Mathematics, Vedant College of Engineering and Technology (Rajasthan Technical University), \\ Bundi 323021, Rajasthan, India \\ * Correspondence: iki@wku.ac.kr
}

Received: 30 June 2019; Accepted: 20 July 2019; Published: 26 July 2019

check for updates

\begin{abstract}
In the theory of generalized hypergeometric functions, classical summation theorems for the series ${ }_{2} F_{1},{ }_{3} F_{2},{ }_{4} F_{3},{ }_{5} F_{4}$ and ${ }_{7} F_{6}$ play a key role. Very recently, Masjed-Jamei and Koepf established generalizations of the above-mentioned summation theorems. Inspired by their work, the main objective of the paper is to provide a new class of Laplace-type integrals involving generalized hypergeometric functions ${ }_{p} F_{p}$ for $p=2,3,4,5$ and 7 in the most general forms. Several new and known cases have also been obtained as special cases of our main findings.
\end{abstract}

Keywords: generalized hypergeometric functions; classical summation theorems; generalization; laplace transforms

MSC: 33C20; 33C05; 33C90

\section{Introduction}

The generalized hypergeometric function with $p$ numerator and $q$ denominator parameters is defined [1-4] as

$$
{ }_{p} F_{q}\left[\begin{array}{lll}
a_{1}, & \cdots, & a_{p} \\
b_{1}, & \cdots, & b_{q}
\end{array}\right]=\sum_{n=0}^{\infty} \frac{\left(a_{1}\right)_{n} \cdots\left(a_{p}\right)_{n}}{\left(b_{1}\right)_{n} \cdots\left(b_{q}\right)_{n}} \frac{z^{n}}{n !},
$$

in which no denominator parameters $b_{j}$ is allowed to be zero or a negative integer. If any numerator parameter $a_{j}$ in Equation (1) is zero or a negative integer, the series terminates.

In addition, here, $(a)_{n}$ is the well known Pochhammer symbol [5] for any complex number $a$ defined as

$$
\begin{aligned}
(a)_{n} & =\frac{\Gamma(a+n)}{\Gamma(a)} \\
& = \begin{cases}1, & (n=0, a \in \mathbb{C} \backslash\{0\}) \\
a(a+1) \cdots(a+n-1), & (n \in \mathbb{N}, a \in \mathbb{C}),\end{cases}
\end{aligned}
$$

where $\Gamma(z)$ is the well known gamma function defined by

$$
\Gamma(z)=\int_{0}^{\infty} e^{-x} x^{z-1} d x
$$

for $\operatorname{Re}(z)>0$.

Further, application of the ratio test shows that the series in Equation (1): 
(i) converges for all finite $z$ if $p \leq q$;

(ii) converges for $|z|<1$ if $p=q+1$; and

(iii) diverges for all $z z \neq 0$ if $p>q+1$.

In addition, following Bromwich [6] (p. 41 and 241), Knopp [7] (p. 401) or Luke [8], it can be shown that the ${ }_{q+1} F_{q}$ series is

(i) absolutely convergent for $|z|=1$ if $\operatorname{Re}(\eta)<0$;

(ii) conditionally convergent for $|z|=1, z \neq 1$ if $0 \leq \operatorname{Re}(\eta)<1$; and

(iii) divergent for $|z|=1$ if $1 \leq \operatorname{Re}(\eta)$, where

$$
\eta=\sum_{j=1}^{p} a_{j}-\sum_{j=1}^{q} b_{j} .
$$

It is not out of place to mention here that, whenever a generalized hypergeometric function reduces to products and quotients of gamma functions, the results are very useful from the point of view of applications. For $p=2,3,4,5$ and 7 of the generalized hypergeometric function in Equation (1) with proper choice of parameters, the results in the form of summation theorems are available in the literature in terms of gamma function. However, for $p=6$, we do not have any summation theorem available. Thus, in this paper, we do not consider the case for $p=6$. Here, we mention the following classical summation theorems $[1,2]$, so that the paper may be self contained.

- Gauss Theorem for $\operatorname{Re}(c-a-b)>0$

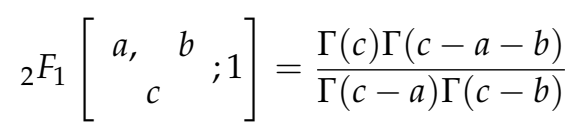

- Kummer's Theorem

$$
{ }_{2} F_{1}\left[\begin{array}{c}
a, b \\
1+a-b
\end{array} ;-1\right]=\frac{\Gamma(1+a-b) \Gamma\left(1+\frac{1}{2} a\right)}{\Gamma\left(1-b+\frac{1}{2} a\right) \Gamma(1+a)}
$$

- $\quad$ Second Gauss Theorem

$$
{ }_{2} F_{1}\left[\begin{array}{c}
a, b \\
\frac{1}{2}(a+b+1)
\end{array} ; \frac{1}{2}\right]=\frac{\sqrt{\pi} \Gamma\left(\frac{1}{2}(a+b+1)\right)}{\Gamma\left(\frac{1}{2}(a+1)\right) \Gamma\left(\frac{1}{2}(b+1)\right)}
$$

- $\quad$ Bailey's Theorem

$$
{ }_{2} F_{1}\left[\begin{array}{ll}
a, & 1-a \\
b
\end{array} ; \frac{1}{2}\right]=\frac{\Gamma\left(\frac{1}{2} b\right) \Gamma\left(\frac{1}{2}(b+1)\right)}{\Gamma\left(\frac{1}{2}(a+b)\right) \Gamma\left(\frac{1}{2}(b-a+1)\right)}
$$

- Dixon's Theorem for $\operatorname{Re}(a-2 b-2 c)>-2$

$$
\begin{aligned}
& { }_{3} F_{2}\left[\begin{array}{cc}
a, \quad b, & c \\
1+a-b, & 1+a-c
\end{array} ; 1\right] \\
& =\frac{\Gamma\left(1+\frac{1}{2} a\right) \Gamma(1+a-b) \Gamma(1+a-c) \Gamma\left(1-b-c+\frac{1}{2} a\right)}{\Gamma(1+a) \Gamma\left(1-b+\frac{1}{2} a\right) \Gamma\left(1-c+\frac{1}{2} a\right) \Gamma(1+a-b-c)}
\end{aligned}
$$

- Watson's Theorem for $\operatorname{Re}(2 c-a-b)>1$

$$
\begin{aligned}
& { }_{3} F_{2}\left[\begin{array}{ccc}
a, b, & c \\
\frac{1}{2}(a+b+1), & 2 c
\end{array}\right] \\
& =\frac{\sqrt{\pi} \Gamma\left(c+\frac{1}{2}\right) \Gamma\left(\frac{1}{2}(a+b+1)\right) \Gamma\left(c-\frac{1}{2}(a+b-1)\right)}{\Gamma\left(\frac{1}{2}(a+1)\right) \Gamma\left(\frac{1}{2}(b+1)\right) \Gamma\left(c-\frac{1}{2}(a-1)\right) \Gamma\left(c-\frac{1}{2}(b-1)\right)}
\end{aligned}
$$


- Whipple's Theorem for $\operatorname{Re}(b)>0$

$$
\begin{aligned}
& { }_{3} F_{2}\left[\begin{array}{ccc}
a, & 1-a, & b \\
c, & 2 b-c+1 & ;
\end{array}\right] \\
& =\frac{\pi 2^{1-2 b} \Gamma(c) \Gamma(2 b-c+1)}{\Gamma\left(\frac{1}{2}(a+c)\right) \Gamma\left(b+\frac{1}{2}(a-c+1)\right) \Gamma\left(\frac{1}{2}(1-a+c)\right) \Gamma\left(b+1-\frac{1}{2}(a+c)\right)}
\end{aligned}
$$

- Pfaff-Saalschütz Theorem

$$
{ }_{3} F_{2}\left[\begin{array}{cc}
a, \quad b, \quad-n \\
c, \quad 1+a+b-c-n
\end{array} ; 1\right]=\frac{(c-a)_{n}(c-b)_{n}}{(c)_{n}(c-a-b)_{n}}
$$

- $\quad$ Second Whipple's Theorem

$$
{ }_{4} F_{3}\left[\begin{array}{c}
a, \quad 1+\frac{1}{2} a, \quad b, \quad c \\
\frac{1}{2} a, a-b+1, a-c+1
\end{array} ;-1\right]=\frac{\Gamma(a-b+1) \Gamma(a-c+1)}{\Gamma(a+1) \Gamma(a-b-c+1)}
$$

- Dougall's Theorem for $\operatorname{Re}(a-c-d-e)>-1$

$$
\begin{aligned}
& { }_{5} F_{4}\left[\begin{array}{rccc}
a, & 1+\frac{1}{2} a, & c, \quad d, & e \\
\frac{1}{2} a, & a-c+1, & a-d+1, & a-e+1
\end{array}\right] \\
& =\frac{\Gamma(a-c+1) \Gamma(a-d+1) \Gamma(a-e+1) \Gamma(a-c-d-e+1)}{\Gamma(a+1) \Gamma(a-d-e+1) \Gamma(a-c-e+1) \Gamma(a-c-d+1)}
\end{aligned}
$$

- Second Dougall's Theorem

$$
\begin{aligned}
& { }_{7} F_{6}\left[\begin{array}{c}
a, \quad 1+\frac{1}{2} a, \quad b, \quad c, \quad d, \quad 1+2 a-b-c-d+n, \quad-n \\
\frac{1}{2} a, a-b+1, a-c+1, a-d+1, b+c+d-a-n, a+1+n
\end{array} ; 1\right] \\
& =\frac{(a+1)_{n}(a-b-c+1)_{n}(a-b-d+1)_{n}(a-c-d+1)_{n}}{(a+1-b)_{n}(a+1-c)_{n}(a+1-d)_{n}(a+1-b-c-d)_{n}}
\end{aligned}
$$

For very interesting applications of some of the above-mentioned classical summation theorems, we refer a very popular and useful paper by Bailey [9].

In addition, for finite sums of hypergeometric series, if we use the following symbol

$$
\stackrel{(m)}{{ }_{p} F_{q}}\left[\begin{array}{lll}
a_{1}, & \cdots, & a_{p} \\
b_{1}, & \cdots, & b_{q}
\end{array}\right]=\sum_{n=0}^{m} \frac{\prod_{i=1}^{p}\left(a_{i}\right)_{n}}{\prod_{i=1}^{q}\left(b_{i}\right)_{n}} \frac{z^{n}}{n !}
$$

where, for instance,

$$
{ }_{p}^{(-1)} F_{q}(z)=0, \quad \stackrel{(0)}{p} F_{q}(z)=1, \quad \stackrel{(1)}{p} F_{q}(z)=1+\frac{a_{1} \cdots a_{p}}{b_{1} \cdots b_{q}} z
$$


then, by using the following relation [10],

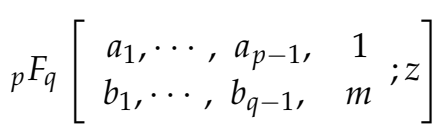

$$
\begin{aligned}
& =\frac{\Gamma\left(b_{1}\right) \cdots \Gamma\left(b_{q-1}\right)}{\Gamma\left(a_{1}\right) \cdots \Gamma\left(a_{p-1}\right)} \frac{\Gamma\left(a_{1}-m+1\right) \cdots \Gamma\left(a_{p-1}-m+1\right)}{\Gamma\left(b_{1}-m+1\right) \cdots \Gamma\left(b_{q-1}-m+1\right)} \frac{(m-1) !}{z^{m-1}} \\
& \times\left\{{ }_{p-1} F_{q-1}\left[\begin{array}{c}
a_{1}-m+1, \cdots, a_{p-1}-m+1 \\
b_{1}-m+1, \cdots, b_{q-1}-m+1
\end{array} ; z\right]\right. \\
& \left.{ }_{-p-1}^{(m-2)} F_{q-1}\left[\begin{array}{l}
a_{1}-m+1, \cdots, a_{p-1}-m+1 \\
b_{1}-m+1, \cdots, b_{q-1}-m+1
\end{array} ; z\right]\right\},
\end{aligned}
$$

very recently Masjed-Jamei and Koepf [11] established generalizations of the classical summation theorems in Equations (4)-(14) in the following form:

$$
\begin{aligned}
& { }_{3} F_{2}\left[\begin{array}{cccc}
a & b & 1 \\
c, & m & ; 1
\end{array}\right] \\
& =\frac{\Gamma(m) \Gamma(c) \Gamma(a-m+1) \Gamma(b-m+1)}{\Gamma(a) \Gamma(b) \Gamma(c-m+1)} \\
& \times\left\{\frac{\Gamma(c-m+1) \Gamma(c-a-b+m-1)}{\Gamma(c-a) \Gamma(c-b)}-{ }_{2}^{(m-2)} F_{1}\left[\begin{array}{c}
a-m+1, b-m+1 \\
c-m+1
\end{array} ; 1\right\}\right. \\
& =\Omega_{1} \\
& { }_{3} F_{2}\left[\begin{array}{c}
a, b, \quad 1 \\
a-b+m, m
\end{array} ;-1\right] \\
& =(-1)^{m-1} \frac{\Gamma(m) \Gamma(a-b+m) \Gamma(a-m+1) \Gamma(b-m+1)}{\Gamma(a) \Gamma(b) \Gamma(a-b+1)} \\
& \times\left\{\frac{\Gamma(a-b+1) \Gamma\left(1+\frac{1}{2}(a-m+1)\right)}{\Gamma(2+a-m) \Gamma\left(m-b+\frac{1}{2}(a-m+1)\right)}-{ }_{2}^{(m-2)} F_{1}\left[\begin{array}{c}
a-m+1, b-m+1 \\
a-b+1
\end{array} ;-1\right]\right\} \\
& =\Omega_{2} \\
& { }_{3} F_{2}\left[\begin{array}{c}
a, b, 1 \\
\frac{1}{2}(a+b+1), m ; \frac{1}{2}
\end{array}\right] \\
& =2^{m-1} \frac{\Gamma(m) \Gamma\left(\frac{1}{2}(a+b+1)\right) \Gamma(a-m+1) \Gamma(b-m+1)}{\Gamma(a) \Gamma(b) \Gamma\left(-m+1+\frac{1}{2}(a+b+1)\right)} \\
& \times\left\{\frac{\sqrt{\pi} \Gamma\left(-m+1+\frac{1}{2}(a+b+1)\right)}{\Gamma\left(1+\frac{1}{2}(a-m)\right) \Gamma\left(1+\frac{1}{2}(b-m)\right)}-{ }_{2}^{(m-2)} F_{1}\left[\begin{array}{c}
a-m+1, b-m+1 \\
-m+1+\frac{1}{2}(a+b+1)
\end{array} ; \frac{1}{2}\right]\right\} \\
& =\Omega_{3}
\end{aligned}
$$




$$
\begin{aligned}
& { }_{3} F_{2}\left[\begin{array}{c}
a, 2 m-a-1,1 \\
b, m
\end{array} ; \frac{1}{2}\right] \\
& =2^{m-1} \frac{\Gamma(m) \Gamma(b) \Gamma(a-m+1) \Gamma(m-a)}{\Gamma(a) \Gamma(2 m-a-1) \Gamma(b-m+1)} \\
& \times\left\{\frac{\Gamma\left(\frac{1}{2}(b-m+1)\right) \Gamma\left(\frac{1}{2}(b-m+2)\right)}{\Gamma\left(-m+1+\frac{1}{2}(a+b)\right) \Gamma\left(\frac{1}{2}(b-a+1)\right)}-{ }_{2}^{(m-2)} F_{1}\left[\begin{array}{c}
a-m+1, m-a \\
b-m+1
\end{array} ; \frac{1}{2}\right]\right\} \\
& =\Omega_{4} \\
& { }_{4} F_{3}\left[\begin{array}{ccc}
a, \quad b, & c, \quad 1 \\
a-b+m, & a-c+m, m
\end{array} ; 1\right] \\
& =\frac{\Gamma(m) \Gamma(a-b+m) \Gamma(a-c+m) \Gamma(a+1-m) \Gamma(b+1-m) \Gamma(c+1-m)}{\Gamma(a) \Gamma(b) \Gamma(c) \Gamma(a-b+1) \Gamma(a-c+1)} \\
& \times\left\{\frac{\Gamma\left(\frac{1}{2}(a+3-m)\right) \Gamma(a-b+1) \Gamma(a-c+1) \Gamma\left(-b-c+\frac{1}{2}(a+3 m-1)\right)}{\Gamma(a+2-m) \Gamma\left(-b+\frac{1}{2}(a+m+1)\right) \Gamma\left(-c+\frac{1}{2}(a+m+1)\right) \Gamma(a-b-c+m)}\right.
\end{aligned}
$$

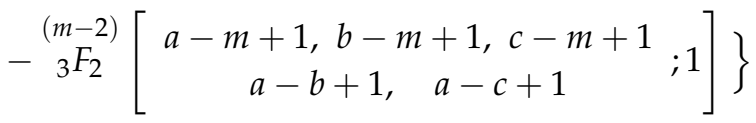

$$
\begin{aligned}
& =\Omega_{5} \\
& \left.{ }_{4} F_{3}\left[\begin{array}{ccc}
a, \quad b, & c, \quad 1 \\
\frac{1}{2}(a+b+1), & 2 c+1-m, m
\end{array}\right] 1\right] \\
& =\frac{\Gamma(m) \Gamma\left(\frac{1}{2}(a+b+1)\right) \Gamma(2 c+1-m) \Gamma(a+1-m) \Gamma(b+1-m) \Gamma(c+1-m)}{\Gamma(a) \Gamma(b) \Gamma(c) \Gamma\left(-m+\frac{1}{2}(a+b+3)\right) \Gamma(2 c-2 m+2)} \\
& \times\left\{\frac{\sqrt{\pi} \Gamma\left(c-m+\frac{3}{2}\right) \Gamma\left(-m+\frac{1}{2}(a+b+3)\right) \Gamma\left(c-\frac{1}{2}(a+b-1)\right)}{\Gamma\left(1+\frac{1}{2}(a-m)\right) \Gamma\left(1+\frac{1}{2}(b-m)\right) \Gamma\left(c+1-\frac{1}{2}(a+m)\right) \Gamma\left(c+1-\frac{1}{2}(b+m)\right)}\right.
\end{aligned}
$$

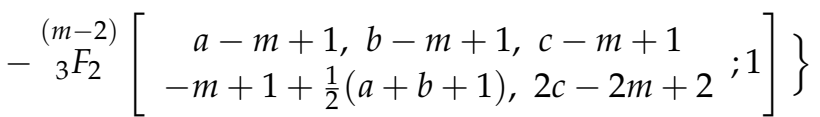

$$
\begin{aligned}
& =\Omega_{6}
\end{aligned}
$$

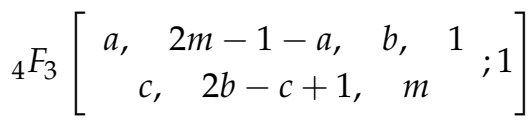

$$
\begin{aligned}
& =\frac{\Gamma(m) \Gamma(c) \Gamma(2 b-c+1) \Gamma(m-a) \Gamma(a+1-m) \Gamma(b+1-m)}{\Gamma(a) \Gamma(b) \Gamma(2 m-1-a) \Gamma(c+1-m) \Gamma(2 b-c-m+2)} \\
& \times\left\{\frac{\pi 2^{2 m-2 b-1} \Gamma(c-m+1)}{\Gamma\left(-m+1+\frac{1}{2}(a+c)\right) \Gamma\left(-m+1+b+\frac{1}{2}(a-c+1)\right) \Gamma\left(\frac{1}{2}(1-a+c)\right)}\right. \\
& \left.\times \frac{\Gamma(2 b-c-m+2)}{\Gamma\left(b+1-\frac{1}{2}(a+c)\right)}-{ }_{3}^{(m-2)} F_{2}\left[\begin{array}{c}
a-m+1, b-m+1, m-a \\
c-m+1,2 b-c-m+2
\end{array} ; 1\right]\right\} \\
& =\Omega_{7}
\end{aligned}
$$




$$
\begin{aligned}
& { }_{4} F_{3}\left[\begin{array}{c}
a, b,-n+m-1,1 \\
c, 1+a+b-c-n, m
\end{array} ; 1\right]=\frac{(m-1) !(1-c)_{m-1}}{(1-a)_{m-1}(1-b)_{m-1}} \\
& \times \frac{(c-a-b+n)_{m-1}}{(n+2-m)_{m-1}} \times\left\{\frac{(c-a)_{n}(c-b)_{n}}{(c+1-m)_{n}(c-a-b+m-1)_{n}}\right. \\
& \left.\quad-{ }_{3}^{(m-2)}\left[\begin{array}{c}
a-m+1, b-m+1,-n \\
c-m+1,2+a+b-c-m-n
\end{array} ; 1\right]\right\} \\
& =\Omega_{8}
\end{aligned}
$$

$$
\begin{aligned}
& { }_{5} F_{4}\left[\begin{array}{c}
a, \quad \frac{1}{2}(a+m+1), \quad b, \quad c, \quad 1 \\
\frac{1}{2}(a+m-1), a-b+m, a-c+m, m
\end{array} ;-1\right]=(-1)^{m-1} \Gamma(m) \\
& \times \frac{\Gamma\left(\frac{1}{2}(a+m-1)\right) \Gamma(a-b+m) \Gamma(a-c+m) \Gamma\left(\frac{1}{2}(a-m+3)\right) \Gamma(a-m+1)}{\Gamma(a) \Gamma(b) \Gamma(c) \Gamma\left(\frac{1}{2}(a+m+1)\right) \Gamma\left(\frac{1}{2}(a-m+1)\right)} \\
& \times \frac{\Gamma(b+1-m) \Gamma(c+1-m)}{\Gamma(a-b+1) \Gamma(a-c+1)} \times\left\{\frac{\Gamma(1+a-b) \Gamma(1+a-c)}{\Gamma(2-m+a) \Gamma(m+a-b-c)}\right. \\
& \left.\quad-{ }_{4}^{(m-2)}\left[\begin{array}{c}
a-m+1, b-m+1, \frac{1}{2}(a-m+3), c-m+1 \\
\frac{1}{2}(a-m+1),
\end{array}\right]\right\} \\
& =\Omega_{9}
\end{aligned}
$$

${ }_{6} F_{5}\left[\begin{array}{ccccc}a, & \frac{1}{2}(a+m+1), & c, \quad d, & e, & 1 \\ \frac{1}{2}(a+m-1), & a-c+m, & a-d+m, & a-e+m, m\end{array}\right]$

$$
\begin{aligned}
& =\frac{\Gamma(m) \Gamma\left(\frac{1}{2}(a+m-1)\right) \Gamma(a-c+m) \Gamma(a-d+m) \Gamma(a-e+m)}{\Gamma(a-c+1) \Gamma(a-d+1) \Gamma(a-e+1)} \\
& \times \frac{\Gamma(a-m+1) \Gamma\left(\frac{1}{2}(a-m+3)\right) \Gamma(c+1-m) \Gamma(d+1-m) \Gamma(e+1-m)}{\Gamma(a) \Gamma(c) \Gamma(d) \Gamma(e) \Gamma\left(\frac{1}{2}(a+m+1)\right) \Gamma\left(\frac{1}{2}(a-m+1)\right)}
\end{aligned}
$$$$
\times\left\{\frac{\Gamma(a-c+1) \Gamma(a-d+1) \Gamma(a-e+1) \Gamma(a-c-d-e+2 m-1)}{\Gamma(2-m+a) \Gamma(a-c-e+m) \Gamma(a-d-e+m) \Gamma(a-c-d+m)}\right.
$$

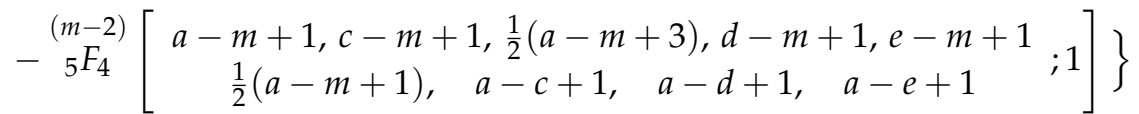

$$
=\Omega_{10}
$$

$$
\begin{aligned}
& { }_{8} F_{7}\left[\begin{array}{c}
a, \quad \frac{1}{2}(a+m+1), \quad b, \quad c, \quad d, \quad 2 a-b-c-d+2 m-1+n, \quad m-n-1, \quad 1 \\
\frac{1}{2}(a+m-1), a-b+m, a-c+m, a-d+m, b+c+d-a+1-m-n, a+n+1, m
\end{array} ; 1\right] \\
& =(-1)^{m-1}(m-1) ! \times \frac{\left(\frac{1}{2}(3-a-m)\right)_{m-1}(1-a+b-m)_{m-1}}{\left(\frac{1}{2}(1-a-m)\right)_{m-1}(1-a)_{m-1}} \\
& \times \frac{(1-a+c-m)_{m-1}(1-a+d-m)_{m-1}(m+n+a-b-c-d)_{m-1}(-a-n)_{m-1}}{(1-b)_{m-1}(1-c)_{m-1}(1-d)_{m-1}(b+c+d-2 a+2-2 m-n)_{m-1}(n+2-m)_{m-1}} \\
& \times\left\{\frac{(a-m+2)_{n}(a-b-c+m)_{n}(a-b-d+m)_{n}(a-c-d+m)_{n}}{(a-b+1)_{n}(a-c+1)_{n}(a-d+1)_{n}(a-b-c-d+2 m-1)_{n}}\right. \\
& \left.-{ }_{7} F_{6}\left[\begin{array}{c}
a-m+1, \frac{1}{2}(a-m+3), b-m+1, c-m+1, d-m+1,2 a-b-c-d+m+n,-n \\
\frac{1}{2}(a-m+1), a-b+1, a-c+1, a-d+1, b+c+d+a+2-2 m-n, a-m+n+2
\end{array} ; 1\right]\right\} \\
& =
\end{aligned}
$$


Remark 1. For other generalizations of the results in Equations (5)-(10), we refer to [12-16].

On the other hand, we define the (direct) Laplace transform of a function $f(t)$ of a real variable $t$ as the integral $g(s)$ over a range of the complex parameter $s$ as

$$
g(s)=\mathcal{L}\{f(t) ; s\}=\int_{0}^{\infty} e^{-s t} f(t) d t
$$

provided the integral exists in the Lebesgue sense. For more details, see, for instance, the works of [17] or [18]. It is interesting to mention here that, in view of the formula

$$
\int_{0}^{\infty} e^{-s t} t^{\alpha-1} d t=\Gamma(\alpha) s^{-\alpha}
$$

provided $\operatorname{Re}(s)>0$ and $\operatorname{Re}(\alpha)>0$, by using Equation (1), it is a simple exercise to show that the Laplace transform of a generalized hypergeometric function ${ }_{p} F_{q}$ is obtained as $[3,19,20]$ :

$$
\begin{aligned}
& \int_{0}^{\infty} e^{-s t} t^{v-1}{ }_{p} F_{q}\left[\begin{array}{lll}
a_{1}, & \cdots, & a_{p} \\
b_{1}, & \cdots, & b_{q}
\end{array}\right] d t \\
& =\Gamma(v) s_{p+1}^{-v} F_{q}\left[\begin{array}{ccc}
v, a_{1}, & \cdots, & a_{p} \\
b_{1}, & \cdots, & b_{q}
\end{array} ;\right.
\end{aligned}
$$

provided that when $p<q, \operatorname{Re}(v)>0, \operatorname{Re}(s)>0$ for $w$ arbitrary, or $p=q>0, \operatorname{Re}(v)>0$ and $\operatorname{Re}(s)>\operatorname{Re}(w)$.

Further, in Equation (29a), if we take $p=q=1, v=b, a_{1}=a$ and $b_{1}=c$, we find that (see [20]),

$$
\int_{0}^{\infty} e^{-s t} t^{b-1}{ }_{1} F_{1}\left[\begin{array}{l}
a \\
c
\end{array} ; w t\right] d t=\Gamma(b) s^{-b}{ }_{2} F_{1}\left[\begin{array}{c}
a, b \\
c
\end{array} ; \frac{w}{s}\right]
$$

provided $\operatorname{Re}(b)>0, \operatorname{Re}(s)>0, \operatorname{Re}(s)>\operatorname{Re}(w)$ and $|s|>|w|$.

Finally, in Equation (29b), if we take $w=\frac{1}{2} s$ and either $c=\frac{1}{2}(a+b+1)$ or $b=1-a$, then it is easy to see that the ${ }_{2} F_{1}$ series appearing on the right-hand side of Equation (29b) are summable by known summation theorems in Equations (6) and (7), respectively, and we find that

$$
\int_{0}^{\infty} e^{-s t} t^{b-1}{ }_{1} F_{1}\left[\begin{array}{c}
a \\
\frac{1}{2}(a+b+1)^{\prime}
\end{array} ; \frac{1}{2} s t\right] d t=s^{-b} \frac{\Gamma\left(\frac{1}{2}\right) \Gamma(b) \Gamma\left(\frac{1}{2}(a+b+1)\right)}{\Gamma\left(\frac{1}{2}(a+1)\right) \Gamma\left(\frac{1}{2}(b+1)\right)}
$$

provided $\operatorname{Re}(b)>0$ and $\operatorname{Re}(s)>0$, and that

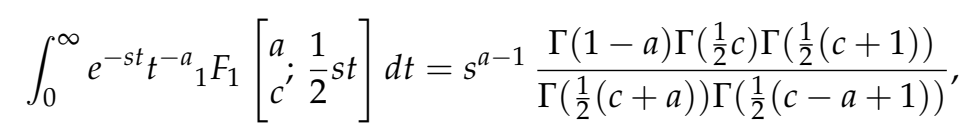

provided $\operatorname{Re}(1-a)>0$ and $\operatorname{Re}(s)>0$.

The results in Equations (29b) and (29c) are very well-known in the literature and are recorded, for example, in the work of [20].

In addition to Equation (29a), it is interesting to observe here that, when $w= \pm s$ and $q=p$, on similar lines, we can obtain the following result in view of the conditions of convergence of ${ }_{p} F_{q}$ mentioned in Section 1.

$$
\begin{aligned}
& \int_{0}^{\infty} e^{-s t} t^{v-1}{ }_{p} F_{p}\left[\begin{array}{lll}
a_{1}, & \cdots, & a_{p} \\
b_{1}, & \cdots, & b_{p}
\end{array} ; s t\right] d t \\
& =\Gamma(v) s_{p+1}^{-v} F_{p}\left[\begin{array}{ccc}
v, a_{1}, & \cdots, & a_{p} \\
b_{1}, & \cdots, & b_{p}
\end{array}\right]
\end{aligned}
$$


provided $\operatorname{Re}(v)>0, \operatorname{Re}(s)>0$ and $\operatorname{Re}\left(b_{1}+\cdots+b_{p}-a_{1}-\cdots-a_{p}-v\right)>0$.

Remark 2. 1. Since there is no summation theorems for the series ${ }_{p} F_{p}$ for argument $\frac{1}{2}, 1$ and -1 are available in the literature, at this moment, it is not possible to find the Laplace-type integrals for the generalized hypergeometric function ${ }_{p} F_{p}$. We leave this open problem for the readers.

2. Laplace-type integrals in the case $p=2$ were given by Deepthi et al. [21] and connections with fractional integral operators were recently studied by Parmar and Purohit [22].

The aim of this paper is to provide a new class of Laplace-type integrals involving generalized hypergeometric functions by employing the summation theorems in Equations (16)-(26). Several new and known special cases have also been considered.

\section{Laplace-Type Integrals Involving Generalized Hypergeometric Functions}

In this section, we establish several new, interesting and elementary Laplace-type integrals in the most general form, involving generalized hypergeometric functions asserted in the following theorems that follow directly from Equations (29a) and (29e) and the known results in Equations (16)-(26). The results presented in this section would serve as key formulas from which, on specializing the parameters, lead to several results, some of which are known and others are believed to be new.

[A] Laplace-type integrals involving generalized hypergeometric function ${ }_{2} F_{2}$

The results to be established are asserted in the following theorems.

Theorem 1. For $m \in \mathbb{N}, \operatorname{Re}(s)>0, \operatorname{Re}(a)>0$ and $\operatorname{Re}(c-a-b+m)>1$, the following result holds true.

$$
\int_{0}^{\infty} e^{-s t} t^{a-1}{ }_{2} F_{2}\left[\begin{array}{ll}
b, & 1 \\
c, & m^{\prime}
\end{array}\right] d t=\Gamma(a) s^{-a} \Omega_{1}
$$

where $\Omega_{1}$ is the same as given in Equation (16).

Theorem 2. For $m \in \mathbb{N}, \operatorname{Re}(s)>0$ and $\operatorname{Re}(c-a-b+m)>1$, the following result holds true.

$$
\int_{0}^{\infty} e^{-s t}{ }_{2} F_{2}\left[\begin{array}{ll}
a, & b \\
c, & m^{\prime}
\end{array}\right] d t=s^{-1} \Omega_{1}
$$

where $\Omega_{1}$ is the same as given in Equation (16).

Theorem 3. For $m \in \mathbb{N}, \operatorname{Re}(s)>0$ and $\operatorname{Re}(a)>0$, the following result holds true.

$$
\int_{0}^{\infty} e^{-s t} t^{a-1}{ }_{2} F_{2}\left[\begin{array}{cc}
b, & 1 \\
a-b+m, & m^{\prime}
\end{array}-s t\right] d t=\Gamma(a) s^{-a} \Omega_{2},
$$

where $\Omega_{2}$ is the same as given in Equation (17).

Theorem 4. For $m \in \mathbb{N}, \operatorname{Re}(s)>0$ and $\operatorname{Re}(b)>0$, the following result holds true.

$$
\int_{0}^{\infty} e^{-s t} t^{b-1}{ }_{2} F_{2}\left[\begin{array}{cc}
a, & 1 \\
a-b+m, m^{\prime}
\end{array} ;-s t\right] d t=\Gamma(b) s^{-b} \Omega_{2}
$$

where $\Omega_{2}$ is the same as given in Equation (17).

Remark 3. The reader should observe that the results given in Theorems 3 and 4 are different but obtained from the same known result in Equation (17). 
Theorem 5. For $m \in \mathbb{N}$ and $\operatorname{Re}(s)>0$, the following result holds true.

$$
\int_{0}^{\infty} e^{-s t}{ }_{2} F_{2}\left[\begin{array}{cc}
a, \quad b \\
a-b+m, & m^{\prime}-s t
\end{array}\right] d t=s^{-1} \Omega_{2}
$$

where $\Omega_{2}$ is the same as given in Equation (17).

Theorem 6. For $m \in \mathbb{N}, \operatorname{Re}(s)>0$ and $\operatorname{Re}(a)>0$, the following result holds true.

$$
\int_{0}^{\infty} e^{-s t} t^{a-1}{ }_{2} F_{2}\left[\begin{array}{cc}
b, & 1 \\
\frac{1}{2}(a+b+1) & m^{\prime}
\end{array} \frac{1}{2} s t\right] d t=\Gamma(a) s^{-a} \Omega_{3},
$$

where $\Omega_{3}$ is the same as given in Equation (18).

Theorem 7. For $m \in \mathbb{N}$ and $\operatorname{Re}(s)>0$, the following result holds true.

$$
\int_{0}^{\infty} e^{-s t}{ }_{2} F_{2}\left[\begin{array}{cc}
a, & b \\
\frac{1}{2}(a+b+1), & m
\end{array} ; \frac{1}{2} s t\right] d t=s^{-1} \Omega_{3},
$$

where $\Omega_{3}$ is the same as given in Equation (18).

Theorem 8. For $m \in \mathbb{N}, \operatorname{Re}(s)>0$ and $\operatorname{Re}(a)>0$, the following result holds true.

$$
\int_{0}^{\infty} e^{-s t} t_{2}^{a-1} F_{2}\left[\begin{array}{crr}
2 m-a-1,1 & 1 \\
b, & m & 2
\end{array}\right] d t=\Gamma(a) s^{-a} \Omega_{4}
$$

where $\Omega_{4}$ is the same as given in Equation (19).

Theorem 9. For $m \in \mathbb{N}, \operatorname{Re}(s)>0$ and $\operatorname{Re}(2 m-a-1)>0$, the following result holds true.

$$
\int_{0}^{\infty} e^{-s t} t^{2 m-a-2}{ }_{2} F_{2}\left[\begin{array}{cc}
a, & 1 \\
b, & m^{\prime}
\end{array} \frac{1}{2} s t\right] d t=\Gamma(2 m-a-1) s^{a+1-2 m} \Omega_{4},
$$

where $\Omega_{4}$ is the same as given in Equation (19).

Theorem 10. For $m \in \mathbb{N}$ and $\operatorname{Re}(s)>0$, the following result holds true.

$$
\int_{0}^{\infty} e^{-s t}{ }_{2} F_{2}\left[\begin{array}{cc}
a, & 2 m-a-1 \\
b, & m
\end{array} ; \frac{1}{2} s t\right] d t=s^{-1} \Omega_{4}
$$

where $\Omega_{4}$ is the same as given in Equation (19).

Proof. To establish the result in Equation (30) asserted in Theorem 1, we proceed as follows. In Equation (29e), if we take $p=q=2, v=a, a_{1}=b, a_{2}=1, b_{1}=c$, and $b_{2}=m$, considering positive sign, we get

$$
\int_{0}^{\infty} e^{-s t} t^{a-1}{ }_{2} F_{2}\left[\begin{array}{cc}
b, & 1 \\
c, & m^{\prime}
\end{array} ; t\right] d x=s^{-a} \Gamma(a)_{3} F_{2}\left[\begin{array}{ccc}
a, & b, & 1 \\
c, & m
\end{array} ; 1\right] .
$$

We now observe that the ${ }_{3} F_{2}$ appearing on the right-hand side of Equation (40) can be evaluated with the help of the result in Equation (16) and we easily arrive at the right-hand side of Equation (30). This completes the proof of Equation (30) asserted in Theorem 1. 
In exactly the same manner, the results in Equations (31)-(39) asserted in Theorems 2-10 can be evaluated. We however omit the details.

Corollary 1. (a) In Theorem 1, if we take $m=1,2,3$, we get the following results.

$$
\begin{aligned}
& \int_{0}^{\infty} e^{-s t} t^{a-1}{ }_{1} F_{1}\left[\begin{array}{l}
b \\
c
\end{array} ; s t\right] d t=\frac{\Gamma(a) \Gamma(c) \Gamma(c-a-b)}{s^{a} \Gamma(c-a) \Gamma(c-b)}, \\
& \int_{0}^{\infty} e^{-s t} t^{a-1}{ }_{2} F_{2}\left[\begin{array}{ll}
b, & 1 \\
c, & 2
\end{array} ; s t\right] d t \\
& =\frac{(c-1) \Gamma(a-1)}{s^{a}(b-1)}\left\{\frac{\Gamma(c-1) \Gamma(c-a-b+1)}{\Gamma(c-a) \Gamma(c-b)}-1\right\}
\end{aligned}
$$

and

$$
\begin{aligned}
& \int_{0}^{\infty} e^{-s t} t^{a-1}{ }_{2} F_{2}\left[\begin{array}{ll}
b, & 1 \\
c, & 3
\end{array} ; s t\right] d t \\
& =\frac{2 \Gamma(a)(c-2)_{2}}{s^{a}(a-2)_{2}(b-2)_{2}}\left\{\frac{\Gamma(c-2) \Gamma(c-a-b+2)}{\Gamma(c-a) \Gamma(c-b)}-\frac{a b+c-2 a-2 b+2}{c-2}\right\} .
\end{aligned}
$$

(b) In Theorem 4, if we take $m=1,2,3$, we get the following results.

$$
\begin{aligned}
& \int_{0}^{\infty} e^{-s t} t^{b-1}{ }_{1} F_{1}\left[\begin{array}{c}
a \\
1+a-b^{\prime}
\end{array}-s t\right] d t=\frac{2^{-a} \Gamma(b) \Gamma\left(\frac{1}{2}\right) \Gamma(1+a-b)}{s^{b} \Gamma\left(\frac{1}{2} a+\frac{1}{2}\right) \Gamma\left(1+\frac{1}{2} a-b\right)},
\end{aligned}
$$

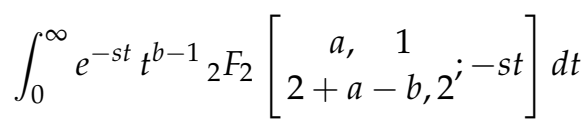

$$
\begin{aligned}
& =\frac{(a-b+1) \Gamma(b)}{s^{b}(a-1)(b-1)}\left\{1-\frac{\Gamma(1+a-b) \Gamma\left(\frac{1}{2} a+\frac{1}{2}\right)}{\Gamma(a) \Gamma\left(\frac{1}{2} a-b+\frac{3}{2}\right)}\right\}
\end{aligned}
$$

and

$$
\begin{aligned}
& \int_{0}^{\infty} e^{-s t} t^{a-1}{ }_{2} F_{2}\left[\begin{array}{c}
b, \\
3+a-b, 3^{\prime}
\end{array} ;-s t\right] d t \\
& =\frac{2(a-b+1)_{2} \Gamma(a)}{s^{b}(a-2)_{2}(b-2)_{2}}\left\{\frac{\Gamma\left(\frac{1}{2} a\right) \Gamma(1+a-b)}{\Gamma(a-1) \Gamma\left(\frac{1}{2} a-b+2\right)}-\frac{3 a+b-a b-3}{1+a-b}\right\} .
\end{aligned}
$$

(c) In Theorem 7, if we take $m=1,2,3$, we get the following results.

$$
\begin{gathered}
\int_{0}^{\infty} e^{-s t} t^{a-1}{ }_{1} F_{1}\left[\begin{array}{c}
b \\
\frac{1}{2}(a+b+1)^{;}
\end{array} \frac{1}{2} s t\right] d t=\frac{\sqrt{\pi} \Gamma(a) \Gamma\left(\frac{1}{2}(a+b+1)\right)}{s^{a} \Gamma\left(\frac{1}{2}(a+1)\right) \Gamma\left(\frac{1}{2}(b+1)\right)}, \\
\int_{0}^{\infty} e^{-s t} t^{a-1}{ }_{2} F_{2}\left[\begin{array}{c}
b, \\
\frac{1}{2}(a+b+1), 2 ; \frac{1}{2} s t
\end{array}\right] d t \\
=\frac{(a+b-1) \Gamma(a-1)}{s^{a}(b-1)}\left\{\frac{\sqrt{\pi} \Gamma\left(\frac{1}{2}(a+b-1)\right)}{\Gamma\left(\frac{1}{2} a\right) \Gamma\left(\frac{1}{2} b\right)}-1\right\}
\end{gathered}
$$


and

$$
\begin{aligned}
& \int_{0}^{\infty} e^{-s t} t^{a-1}{ }_{2} F_{2}\left[\begin{array}{c}
b, 1 \\
\frac{1}{2}(a+b+1), 3
\end{array} ; \frac{1}{2} s t\right] d t=\frac{2 \Gamma(a)(a+b-1)(a+b-3)}{s^{a}(a-2)_{2}(b-2)_{2}} \\
& \times\left\{\frac{\sqrt{\pi} \Gamma\left(\frac{1}{2}(a+b-3)\right)}{\Gamma\left(\frac{1}{2}(a-1)\right) \Gamma\left(\frac{1}{2}(b-1)\right)}-\frac{a b-a-b+1}{a+b-3}\right\} .
\end{aligned}
$$

(d) In Theorem 10, if we take $m=1,2,3$, we get the following results.

$$
\begin{gathered}
\int_{0}^{\infty} e^{-s t} t^{a-1}{ }_{1} F_{1}\left[\begin{array}{c}
1-a \\
b
\end{array} ; \frac{1}{2} s t\right] d t=\frac{\Gamma(a) \Gamma\left(\frac{1}{2} b\right) \Gamma\left(\frac{1}{2}(b+1)\right)}{s^{a} \Gamma\left(\frac{1}{2}(a+b)\right) \Gamma\left(\frac{1}{2}(b-a+1)\right)}, \\
\int_{0}^{\infty} e^{-s t} t^{a-1}{ }_{2} F_{2}\left[\begin{array}{cc}
3-a, & 1 \\
b, & 2
\end{array} \frac{1}{2} s t\right] d t \\
=\frac{2(1-b) \Gamma(a)}{s^{a}(1-a)_{2}}\left\{\frac{\Gamma\left(\frac{1}{2}(b-1)\right) \Gamma\left(\frac{1}{2} b\right)}{\Gamma\left(\frac{1}{2}(a+b)-1\right) \Gamma\left(\frac{1}{2}(b-a+1)\right)}-1\right\}
\end{gathered}
$$

and

$$
\begin{aligned}
& \int_{0}^{\infty} e^{-s t} t^{a-1}{ }_{2} F_{2}\left[\begin{array}{cc}
5-a, & 1 \\
b, & 3
\end{array} \frac{1}{2} s t\right] d t=\frac{8(b-2)_{2} \Gamma(a)}{s^{a}(a-4)_{4}} \\
& \times\left\{\frac{\Gamma\left(\frac{1}{2}(b-1)\right) \Gamma\left(\frac{1}{2}(b-2)\right)}{\Gamma\left(\frac{1}{2}(a+b)-2\right) \Gamma\left(\frac{1}{2}(b-a+1)\right)}-\frac{5 a-a^{2}+2 b-10}{2(b-2)}\right\} .
\end{aligned}
$$

Similarly, other results can be obtained from Theorems 2,3,5, 6, 8 and 9 .

Remark 4. 1. The results in Equations (47) and (50) were recorded by [23] as well as [20].

2. The proofs of Theorems 11-35 given below are straight forward and can be proven with the help of the result in Equation (29e), thus they are given here without proof.

[B] Laplace-type integrals involving generalized hypergeometric function ${ }_{3} F_{3}$

The results to be established are asserted in the following theorems.

Theorem 11. For $m \in \mathbb{N}, \operatorname{Re}(s)>0, \operatorname{Re}(a)>0$ and $\operatorname{Re}(a-2 b-2 c+3 m)>1$, the following result holds true.

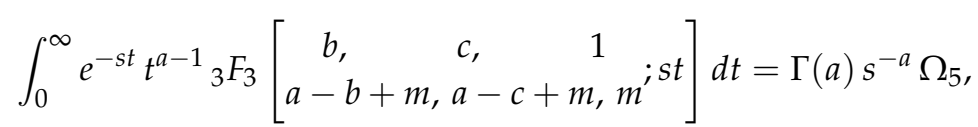

where $\Omega_{5}$ is the same as given in Equation (20).

Theorem 12. For $m \in \mathbb{N}, \operatorname{Re}(s)>0, \operatorname{Re}(b)>0$ and $\operatorname{Re}(a-2 b-2 c+3 m)>1$, the following result holds true.

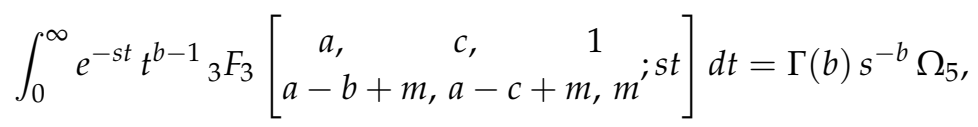

where $\Omega_{5}$ is the same as given in Equation (20). 
Theorem 13. For $m \in \mathbb{N}, \operatorname{Re}(s)>0$ and $\operatorname{Re}(a-2 b-2 c+3 m)>1$, the following result holds true.

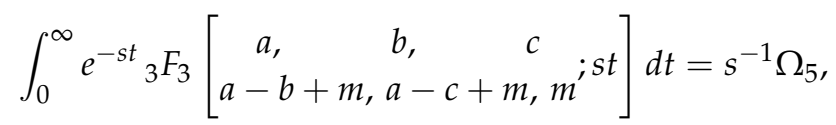

where $\Omega_{5}$ is the same as given in Equation (20).

Theorem 14. For $m \in \mathbb{N}, \operatorname{Re}(s)>0, \operatorname{Re}(a)>0$ and $\operatorname{Re}(2 c-a-b)>-1$, the following result holds true.

$$
\int_{0}^{\infty} e^{-s t} t^{a-1}{ }_{3} F_{3}\left[\begin{array}{cc}
b, & c, \\
\frac{1}{2}(a+b+1), & 2 c+1-m, m^{;} s t
\end{array}\right] d t=\Gamma(a) s^{-a} \Omega_{6}
$$

where $\Omega_{6}$ is the same as given in Equation (21).

Theorem 15. For $m \in \mathbb{N}, \operatorname{Re}(s)>0, \operatorname{Re}(c)>0$ and $\operatorname{Re}(2 c-a-b)>-1$, the following result holds true.

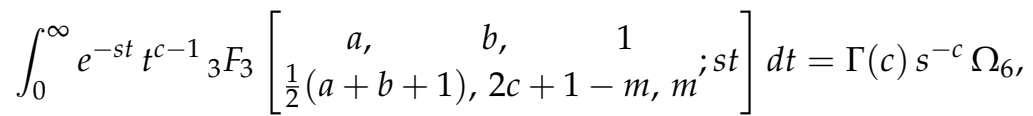

where $\Omega_{6}$ is the same as given in Equation (21).

Theorem 16. For $m \in \mathbb{N}, \operatorname{Re}(s)>0$ and $\operatorname{Re}(2 c-a-b)>-1$, the following result holds true.

$$
\int_{0}^{\infty} e^{-s t}{ }_{3} F_{3}\left[\begin{array}{ccc}
a, & b, & c \\
\frac{1}{2}(a+b+1), & 2 c+1-m, & m
\end{array}\right] d t=s^{-1} \Omega_{6},
$$

where $\Omega_{6}$ is the same as given in Equation (21).

Theorem 17. For $m \in \mathbb{N}, \operatorname{Re}(s)>0, \operatorname{Re}(a)>0$ and $\operatorname{Re}(b-m+1)>0$, the following result holds true.

$$
\int_{0}^{\infty} e^{-s t} t^{a-1}{ }_{3} F_{3}\left[\begin{array}{ccc}
2 m-a-1, & b, & 1 \\
c, & 2 b-c+1, & m^{\prime}
\end{array}\right] d t=\Gamma(a) s^{-a} \Omega_{7},
$$

where $\Omega_{7}$ is the same as given in Equation (22).

Theorem 18. For $m \in \mathbb{N}, \operatorname{Re}(s)>0, \operatorname{Re}(b)>0$ and $\operatorname{Re}(b-m+1)>0$, the following result holds true.

$$
\int_{0}^{\infty} e^{-s t} t^{b-1}{ }_{3} F_{3}\left[\begin{array}{lll}
a, & 2 m-a-1, & 1 \\
c, & 2 b-c+1, & m^{;}
\end{array}\right] d t=\Gamma(b) s^{-b} \Omega_{7},
$$

where $\Omega_{7}$ is the same as given in Equation (22).

Theorem 19. For $m \in \mathbb{N}, \operatorname{Re}(s)>0, \operatorname{Re}(2 m-a-1)>0$ and $\operatorname{Re}(b-m+1)>0$, the following result holds true.

$$
\int_{0}^{\infty} e^{-s t} t^{2 m-a-2}{ }_{3} F_{3}\left[\begin{array}{ccc}
a, & c, & 1 \\
c, & 2 b-c+1, & m^{;}
\end{array}\right] d t=\Gamma(2 m-a-1) s^{a+1-2 m} \Omega_{7},
$$

where $\Omega_{7}$ is the same as given in Equation (22). 
Theorem 20. For $m \in \mathbb{N}, \operatorname{Re}(s)>0$ and $\operatorname{Re}(b-m+1)>0$, the following result holds true.

$$
\int_{0}^{\infty} e^{-s t}{ }_{3} F_{3}\left[\begin{array}{lll}
a, & 2 m-a-1, & b \\
c, & 2 b-c+1, & m
\end{array} ; s t\right] d t=s^{-1} \Omega_{7},
$$

where $\Omega_{7}$ is the same as given in Equation (22).

Theorem 21. For $m \in \mathbb{N}, n \in \mathbb{N}_{0}, \operatorname{Re}(s)>0$ and $\operatorname{Re}(a)>0$, the following result holds true.

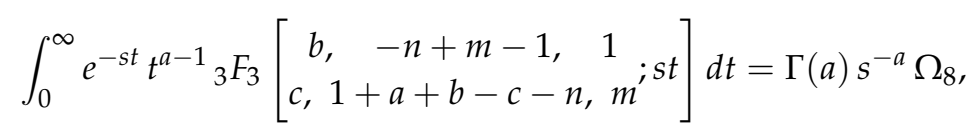

where $\Omega_{8}$ is the same as given in Equation (23).

Theorem 22. For $m \in \mathbb{N}, n \in \mathbb{N}_{0}$ and $\operatorname{Re}(s)>0$, the following result holds true.

$$
\int_{0}^{\infty} e^{-s t}{ }_{3} F_{3}\left[\begin{array}{cc}
a, \quad b, & -n+m-1 \\
c, 1+a+b-c-n, m^{\prime}
\end{array} ; s t\right] d t=s^{-1} \Omega_{8},
$$

where $\Omega_{8}$ is the same as given in Equation (23).

Corollary 2. (e) In Theorem 11, if we take $m=1,2,3$, we get the following results.

$$
\begin{aligned}
& \int_{0}^{\infty} e^{-s t} t^{a-1}{ }_{2} F_{2}\left[\begin{array}{cc}
b, & c \\
1+a-b, & 1+a-c
\end{array} ; s t\right] d t \\
& =\frac{\Gamma\left(\frac{1}{2} a\right) \Gamma(1+a-b) \Gamma(1+a-c) \Gamma\left(1+\frac{1}{2} a-b-c\right)}{2 s^{a} \Gamma\left(1+\frac{1}{2} a-b\right) \Gamma\left(1+\frac{1}{2} a-c\right) \Gamma(1+a-b-c)}, \\
& \int_{0}^{\infty} e^{-s t} t^{a-1}{ }_{3} F_{3}\left[\begin{array}{ccc}
b, & c, & 1 \\
a-b+2, & a-c+2, & 2
\end{array} ; s t\right] d t \\
& =\frac{\Gamma(a-1)(1+a-b)(1+a-c)}{s^{a}(b-1)(c-1)} \\
& \times\left\{\frac{\Gamma\left(\frac{1}{2}(a+1)\right) \Gamma(1+a-b) \Gamma(1+a-c) \Gamma\left(\frac{1}{2} a-b-c+\frac{5}{2}\right)}{\Gamma(a) \Gamma\left(\frac{1}{2} a-b+\frac{3}{2}\right) \Gamma\left(\frac{1}{2} a-c+\frac{3}{2}\right) \Gamma(2+a-b-c)}-1\right\}
\end{aligned}
$$

and

$$
\begin{aligned}
\int_{0}^{\infty} e^{-s t} t^{a-1}{ }_{3} F_{3}\left[\begin{array}{c}
b, \\
a-b+3,
\end{array} \begin{array}{c}
c \\
a-c+3,3
\end{array} ; s t\right] d t \\
=\frac{2(a-b+1)_{2}(a-c+1)_{2} \Gamma(a)}{s^{a}(a-2)_{2}(b-2)_{2}(c-2)_{2}} \\
\quad \times\left\{\frac{\Gamma\left(\frac{1}{2} a\right) \Gamma(1+a-b) \Gamma(1+a-c) \Gamma\left(\frac{1}{2} a-b-c+4\right)}{\Gamma(a-1) \Gamma\left(\frac{1}{2} a-b+2\right) \Gamma\left(\frac{1}{2} a-c+2\right) \Gamma(3+a-b-c)}\right. \\
\left.\quad-\frac{(a-2)(b-2)(c-2)}{(a-b+1)(a-c+1)}-1\right\} .
\end{aligned}
$$


(f) In Theorem 14, if we take $m=1,2,3$, we get the following results.

$$
\begin{gathered}
\int_{0}^{\infty} e^{-s t} t^{a-1}{ }_{2} F_{2}\left[\begin{array}{c}
b, \\
\frac{1}{2}(a+b+1), 2 c
\end{array} ; s t\right] d t \\
=\frac{\sqrt{\pi} \Gamma(a) \Gamma\left(c+\frac{1}{2}\right) \Gamma\left(\frac{1}{2}(a+b+1)\right) \Gamma\left(c-\frac{1}{2}(a+b-1)\right)}{s^{a} \Gamma\left(\frac{1}{2}(a+1) \Gamma\left(\frac{1}{2}(b+1)\right) \Gamma\left(c-\frac{1}{2}(a-1)\right) \Gamma\left(c-\frac{1}{2}(b-1)\right)\right.}, \\
\int_{0}^{\infty} e^{-s t} t^{a-1}{ }_{3} F_{3}\left[\begin{array}{c}
b, \quad c, \quad 1 \\
\frac{1}{2}(a+b+1), 2 c-1,2
\end{array} ; s t\right] d t \\
=\frac{(a+b-1) \Gamma(a-1)}{s^{a}(b-1)}\left\{\frac{\sqrt{\pi} \Gamma\left(c-\frac{1}{2}\right) \Gamma\left(\frac{1}{2}(a+b-1)\right) \Gamma\left(c-\frac{1}{2}(a+b-1)\right)}{\Gamma\left(\frac{1}{2} a\right) \Gamma\left(\frac{1}{2} b\right) \Gamma\left(c-\frac{1}{2} a\right) \Gamma\left(c-\frac{1}{2} b\right)}-1\right\}
\end{gathered}
$$

and

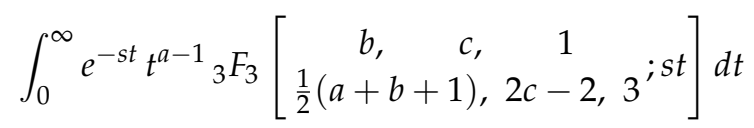

$$
\begin{aligned}
& =\frac{(2 c-3)(a+b-1)(a+b-3) \Gamma(a)}{s^{a}(c-1)(a-2)_{2}(b-2)_{2}} \\
& \times\left\{\frac{\sqrt{\pi} \Gamma\left(c-\frac{3}{2}\right) \Gamma\left(\frac{1}{2}(a+b-3)\right) \Gamma\left(c-\frac{1}{2}(a+b-1)\right)}{\Gamma\left(\frac{1}{2}(a-1)\right) \Gamma\left(\frac{1}{2}(b-1)\right) \Gamma\left(c-\frac{1}{2}(a+1)\right) \Gamma\left(c-\frac{1}{2}(b+1)\right)}\right. \\
& \left.-\frac{(a-2)(b-2)}{a+b-3}-1\right\} \text {. }
\end{aligned}
$$

(g) In Theorem 17, if we take $m=1,2,3$, we get the following results.

$$
\begin{aligned}
& \int_{0}^{\infty} e^{-s t} t^{a-1}{ }_{2} F_{2}\left[\begin{array}{cc}
1-a, & b \\
c, 2 b-c+1
\end{array} ; s t\right] d t \\
& =\frac{\pi 2^{1-2 b} \Gamma(a) \Gamma(c) \Gamma(2 b-c+1)}{s^{a} \Gamma\left(\frac{1}{2}(a+c)\right) \Gamma\left(b+\frac{1}{2}(a-c+1)\right) \Gamma\left(\frac{1}{2}(1-a+c)\right) \Gamma\left(b+1-\frac{1}{2}(a+c)\right)},
\end{aligned}
$$

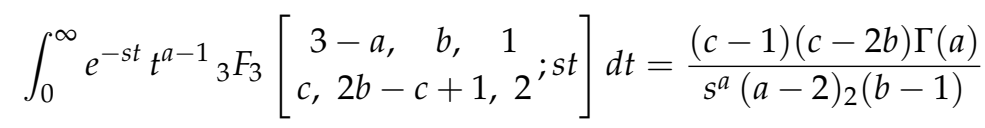

$$
\begin{aligned}
& \times\left\{\frac{\pi 2^{3-2 b} \Gamma(c-1) \Gamma(2 b-c)}{\Gamma\left(\frac{1}{2}(a+c)-1\right) \Gamma\left(b+\frac{1}{2}(a-c-1)\right) \Gamma\left(\frac{1}{2}(1-a+c)\right) \Gamma\left(b+1-\frac{1}{2}(a+c)\right)}-1\right\}
\end{aligned}
$$

and

$$
\begin{aligned}
& \int_{0}^{\infty} e^{-s t} t^{a-1}{ }_{3} F_{3}\left[\begin{array}{cc}
5-a, b, & 1 \\
c, 2 b-c+1,3 & 3
\end{array}\right] d t=\frac{2(c-2)_{2}(2 b-c-1)_{2} \Gamma(a)}{s^{a}(a-4)_{4}(b-2)_{2}} \\
\times & \left\{\frac{\pi 2^{5-2 b} \Gamma(c-2) \Gamma(2 b-c+1)}{\Gamma\left(\frac{1}{2}(a+c)-2\right) \Gamma\left(b+\frac{1}{2}(a-c-3)\right) \Gamma\left(\frac{1}{2}(1-a+c)\right) \Gamma\left(b+1-\frac{1}{2}(a+c)\right)}\right. \\
& \left.-\frac{(a-2)(3-a)(b-2)}{(c-2)(2 b-c-1)}-1\right\} .
\end{aligned}
$$


(h) In Theorem 21, if we take $m=1,2,3$, we get the following results.

$$
\begin{gathered}
\int_{0}^{\infty} e^{-s t} t^{a-1}{ }_{2} F_{2}\left[\begin{array}{c}
-n, \quad b \\
1+a+b-c-n, c
\end{array} ; s t\right] d t=\frac{\Gamma(a)(c-a)_{n}(c-b)_{n}}{s^{a}(c)_{n}(c-a-b)_{n}}, \\
\quad \int_{0}^{\infty} e^{-s t} t^{a-1}{ }_{3} F_{3}\left[\begin{array}{c}
-n+1, \quad b, \\
1+a+b-c-n, c, 2
\end{array} ; s t\right] d t \\
=\frac{(1-c)(c-a-b+n) \Gamma(a-1)}{n(1-b) s^{a}}\left\{\frac{(c-a)_{n}(c-b)_{n}}{(c)_{n}(c-a-b+1)_{n}}-1\right\}
\end{gathered}
$$

and

$$
\begin{aligned}
& \int_{0}^{\infty} e^{-s t} t^{a-1}{ }_{3} F_{3}\left[\begin{array}{ccc}
-n+2, & b, & 1 \\
1+a+b-c-n, c, 3
\end{array} ; s t\right] d t \\
& =\frac{2(1-c)_{2}(c-a-b+n)_{2} \Gamma(a)}{s^{a}(1-a)_{2}(1-b)_{2}} \\
& \times\left\{\frac{(c-a)_{n}(c-b)_{n}}{(c-2)_{n}(c-a-b+2)_{n}}+\frac{n(a-2)(b-2)}{(c-2)(a+b-c-n-1)}-1\right\} .
\end{aligned}
$$

Remark 5. The results in Equations (65), (68) and (71) are known results due to Kim et al. [24].

[C] Laplace-type integrals involving generalized hypergeometric function ${ }_{4} F_{4}$

The results to be established are asserted in the following theorems.

Theorem 23. For $m \in \mathbb{N}, \operatorname{Re}(s)>0, \operatorname{Re}(a)>0$ and $\operatorname{Re}(a-2 b-2 c+3 m)>2$, the following result holds true.

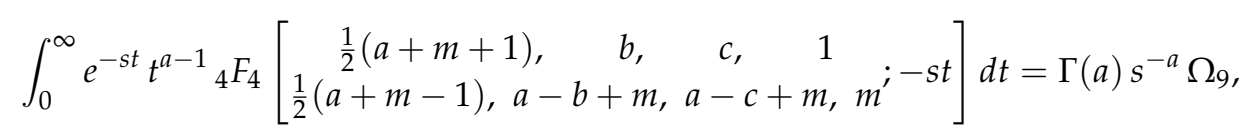

where $\Omega_{9}$ is the same as given in Equation (24).

Theorem 24. For $m \in \mathbb{N}, \operatorname{Re}(s)>0, \operatorname{Re}(c)>0$ and $\operatorname{Re}(a-2 b-2 c+3 m)>2$, the following result holds true.

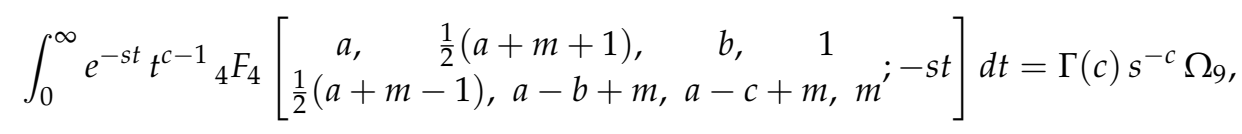

where $\Omega_{9}$ is the same as given in Equation (24).

Theorem 25. For $m \in \mathbb{N}, \operatorname{Re}(s)>0, \operatorname{Re}(a+m+1)>0$ and $\operatorname{Re}(a-2 b-2 c+3 m)>2$, the following result holds true.

$$
\begin{aligned}
& \int_{0}^{\infty} e^{-s t} t^{\frac{1}{2}(a+m-1)}{ }_{4} F_{4}\left[\begin{array}{cccc}
a, & b, & c, & 1 \\
\frac{1}{2}(a+m-1), & a-b+m, & a-c+m, & m^{\prime}-s t
\end{array}\right] d t \\
& =\Gamma\left(\frac{1}{2}(a+m+1)\right) s^{-\frac{1}{2}(a+m+1)} \Omega_{9},
\end{aligned}
$$

where $\Omega_{9}$ is the same as given in Equation (24). 
Theorem 26. For $m \in \mathbb{N}, \operatorname{Re}(s)>0$ and $\operatorname{Re}(a-2 b-2 c+3 m)>2$, the following result holds true.

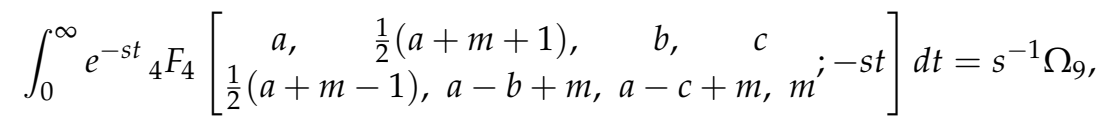

where $\Omega_{9}$ is the same as given in Equation (24).

Corollary 3. (i) In Theorem 24 , if we take $m=1,2,3$, we get the following results.

$$
\begin{aligned}
& \int_{0}^{\infty} e^{-s t} t^{c-1}{ }_{3} F_{3}\left[\begin{array}{cc}
a, & \frac{1}{2}(a+2), \quad b \\
\frac{1}{2} a, & a-b+1, a-c+1
\end{array} ;-s t\right] d t \\
& =\frac{\Gamma(1+a-b) \Gamma(1+a-c) \Gamma(c)}{s^{c} \Gamma(1+a) \Gamma(1+a-b-c)}, \\
& \int_{0}^{\infty} e^{-s t} t^{c-1}{ }_{4} F_{4}\left[\begin{array}{ccc}
a, & \frac{1}{2}(a+3), & b, \\
\frac{1}{2}(a+1), & a-b+2, & a-c+2,2
\end{array} ;-s t\right] d t \\
& =\frac{(1+a-b)(1+a-c) \Gamma(c)}{s^{c}(a+1)(b-1)(c-1)}\left\{1-\frac{\Gamma(1+a-b) \Gamma(1+a-c)}{\Gamma(a) \Gamma(2+a-b-c)}\right\}
\end{aligned}
$$

and

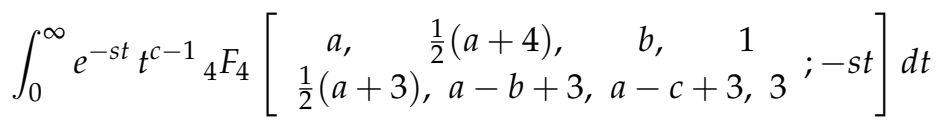

$$
\begin{aligned}
& =\frac{2(1+a-b)_{2}(1+a-c)_{2} \Gamma(c)}{s^{c}(a+2)(a-1)(b-2)_{2}(c-2)_{2}} \\
& \times\left\{\frac{\Gamma(1+a-b) \Gamma(1+a-c)}{\Gamma(a-1) \Gamma(3+a-b-c)}+\frac{a(b-2)(c-2)}{(1+a-b)(1+a-c)}-1\right\} .
\end{aligned}
$$

Similarly, other results can be obtained from Theorems 23, 25 and 26.

[D] Laplace-type integrals involving generalized hypergeometric function ${ }_{5} F_{5}$

The results to be established are asserted in the following theorems.

Theorem 27. For $m \in \mathbb{N}, \operatorname{Re}(s)>0, \operatorname{Re}(a)>0$ and $\operatorname{Re}(2 a-2 c-2 d-2 e+3 m)>2$, the following result holds true.

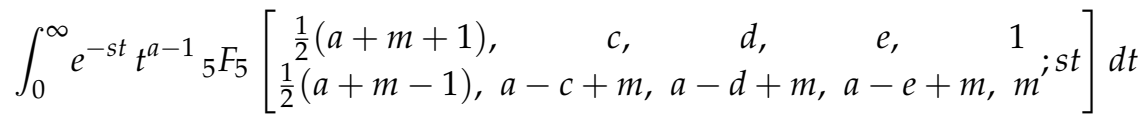

$$
\begin{aligned}
& =\Gamma(a) s^{-a} \Omega_{10} \text {, }
\end{aligned}
$$

where $\Omega_{10}$ is the same as given in Equation (25).

Theorem 28. For $m \in \mathbb{N}, \operatorname{Re}(s)>0, \operatorname{Re}(c)>0$ and $\operatorname{Re}(2 a-2 c-2 d-2 e+3 m)>2$, the following result holds true.

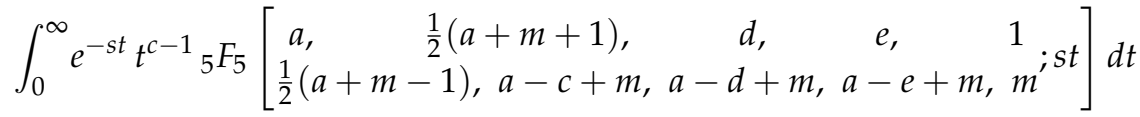

$$
\begin{aligned}
& =\Gamma(c) s^{-c} \Omega_{10},
\end{aligned}
$$

where $\Omega_{10}$ is the same as given in Equation (25). 
Theorem 29. For $m \in \mathbb{N}, \operatorname{Re}(s)>0, \operatorname{Re}(a+m+1)>0$ and $\operatorname{Re}(2 a-2 c-2 d-2 e+3 m)>2$, the following result holds true.

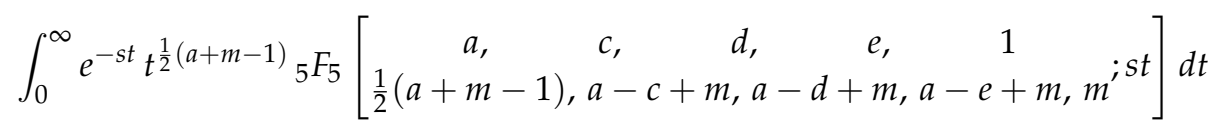

$$
\begin{aligned}
& =\Gamma\left(\frac{1}{2}(a+m+1)\right) s^{-\frac{1}{2}(a+m+1)} \Omega_{10},
\end{aligned}
$$

where $\Omega_{10}$ is the same as given in Equation (25).

Theorem 30. For $m \in \mathbb{N}, \operatorname{Re}(s)>0$ and $\operatorname{Re}(2 a-2 c-2 d-2 e+3 m)>2$, the following result holds true.

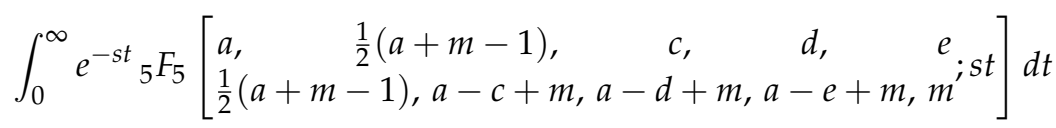

$$
\begin{aligned}
& =s^{-1} \Omega_{10} \text {, }
\end{aligned}
$$

where $\Omega_{10}$ is the same as given in Equation (25).

Corollary 4. (j) In Theorem 27, if we take $m=1,2,3$, we get the following results.

$$
\begin{aligned}
& \int_{0}^{\infty} e^{-s t} t^{a-1}{ }_{4} F_{4}\left[\begin{array}{c}
c, \quad \frac{1}{2}(a+2), \quad d, \quad e \\
\frac{1}{2} a, a-c+1, a-d+1, a-e+1
\end{array} ; s t\right] d t \\
& =\frac{\Gamma(1+a-c) \Gamma(1+a-d) \Gamma(1+a-e) \Gamma(1+a-c-d-e)}{s^{a} \Gamma(1+a-d-e) \Gamma(1+a-c-e) \Gamma(1+a-c-d)},
\end{aligned}
$$

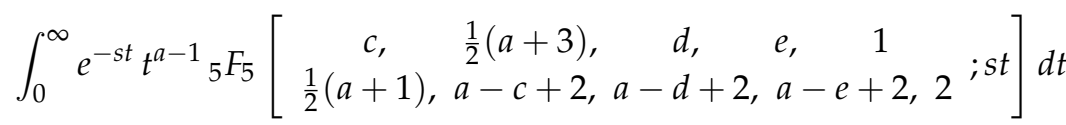

$$
\begin{aligned}
& =\frac{(1+a-c)(1+a-d)(1+a-e) \Gamma(a)}{s^{a}(1+a)(c-1)(d-1)(e-1)} \\
& \times\left\{\frac{\Gamma(1+a-c) \Gamma(1+a-d) \Gamma(1+a-e) \Gamma(3+a-c-d-e)}{\Gamma(a) \Gamma(2+a-d-e) \Gamma(2+a-c-e) \Gamma(2+a-c-d)}-1\right\}
\end{aligned}
$$

and

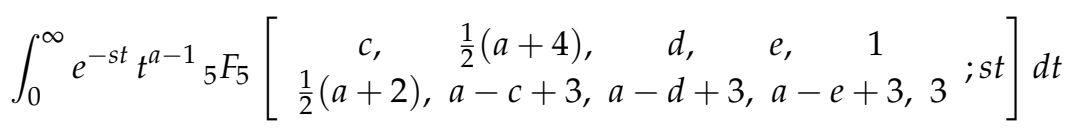

$$
\begin{aligned}
& =\frac{2(1+a-c)_{2}(1+a-d)_{2}(1+a-e)_{2} \Gamma(a)}{s^{a}(a-1)(a+2)(c-2)_{2}(d-2)_{2}(e-2)_{2}} \\
& \times\left\{\frac{\Gamma(1+a-c) \Gamma(1+a-d) \Gamma(1+a-e) \Gamma(5+a-c-d-e)}{\Gamma(a-1) \Gamma(3+a-d-e) \Gamma(3+a-c-e) \Gamma(3+a-c-d)}\right. \\
& \left.-\frac{a(c-2)(d-2)(e-2)}{(1+a-c)(1+a-d)(1+a-e)}\right\} \text {. }
\end{aligned}
$$

Similarly, other results can be obtained from Theorems 28-30.

[E] Laplace-type integrals involving generalized hypergeometric function ${ }_{6} F_{6}$

The results to be established are asserted in the following theorems. 
Theorem 31. For $m \in \mathbb{N}, \operatorname{Re}(s)>0$ and $\operatorname{Re}(a)>0$, the following result holds true.

$$
\begin{aligned}
& \int_{0}^{\infty} e^{-s t} t^{a-1} \times \\
{ }_{7} F_{7} & {\left[\begin{array}{c}
\frac{1}{2}(a+m+1), \quad b, \quad c, \quad d, \quad 2 a-b-c-d+2 m-1+n, \quad m-n-1, \quad 1 \\
\frac{1}{2}(a+m-1), a-b+m, a-c+m, a-d+m, b+c+d-a+1-m-n, a+n+1, m^{\prime}
\end{array} ; t\right] d t } \\
= & \Gamma(a) s^{-a} \Omega_{11},
\end{aligned}
$$

where $\Omega_{11}$ is the same as given in Equation (26).

Theorem 32. For $m \in \mathbb{N}, \operatorname{Re}(s)>0$ and $\operatorname{Re}(a+m+1)>0$, the following result holds true.

$$
\begin{aligned}
& \int_{0}^{\infty} e^{-s t} t^{\frac{1}{2}(a+m-1)} \times \\
& { }_{7} F_{7}\left[\begin{array}{c}
a, \quad b, \quad c, \quad d, \quad 2 a-b-c-d+2 m-1+n, \quad m-n-1, \quad 1 \\
\frac{1}{2}(a+m-1), a-b+m, a-c+m, a-d+m, b+c+d-a+1-m-n, a+n+1, m^{\prime} s t
\end{array}\right] d t \\
= & \Gamma\left(\frac{1}{2}(a+m+1)\right) s^{-\frac{1}{2}(a+m+1)} \Omega_{11},
\end{aligned}
$$

where $\Omega_{11}$ is the same as given in Equation (26).

Theorem 33. For $m \in \mathbb{N}, \operatorname{Re}(s)>0$ and $\operatorname{Re}(b)>0$, the following result holds true.

$$
\begin{aligned}
& \int_{0}^{\infty} e^{-s t} t^{b-1} \times \\
& { }_{7} F_{7}\left[\begin{array}{c}
\frac{1}{2}(a+m+1), \quad b, \quad c, \quad d, \quad 2 a-b-c-d+2 m-1+n, \quad m-n-1, \quad 1 \\
\frac{1}{2}(a+m-1), a-b+m, a-c+m, a-d+m, b+c+d-a+1-m-n, a+n+1, m^{\prime} s t
\end{array} ; d t\right. \\
& =\Gamma(b) s^{-b} \Omega_{11} \text {, }
\end{aligned}
$$

where $\Omega_{11}$ is the same as given in Equation (26).

Theorem 34. For $m \in \mathbb{N}, \operatorname{Re}(s)>0$ and $\operatorname{Re}(2 a-b-c-d+2 m-1+n)>0$, the following result holds true.

$$
\begin{aligned}
& \int_{0}^{\infty} e^{-s t} t^{2 a-b-c-d+2 m+n-2} \times \\
&{ }_{7} F_{7}\left[\begin{array}{cccc}
\frac{1}{2}(a+m+1), & a, \quad b, \quad c, \quad d, \quad m-n-1, & 1 \\
\frac{1}{2}(a+m-1), a-b+m, a-c+m, a-d+m, b+c+d-a+1-m-n, a+n+1, m^{\prime} & s t
\end{array}\right] d t \\
&=\Gamma(2 a-b-c-d+2 m-1+n) s^{-(2 a-b-c-d+2 m-1+n)} \Omega_{11},
\end{aligned}
$$

where $\Omega_{11}$ is the same as given in Equation (26).

Theorem 35. For $m \in \mathbb{N}$ and $\operatorname{Re}(s)>0$, the following result holds true.

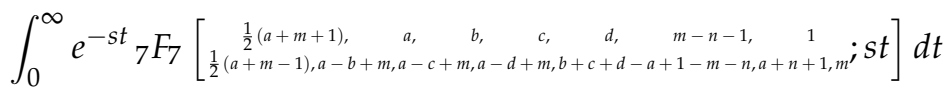

$$
\begin{aligned}
& =s^{-1} \Omega_{11} \text {, }
\end{aligned}
$$

where $\Omega_{11}$ is the same as given in Equation (26). 
Corollary 5. (k) In Theorem 31, if we take $m=1,2,3$, we get the following results.

$$
\begin{aligned}
& \int_{0}^{\infty} e^{-s t} t^{a-1} \\
\times & { }_{6} F_{6}\left[\begin{array}{ccc}
b, & \frac{1}{2}(a+2), \quad c, \quad d, \quad 2 a-b-c-d+n+1, \quad-n \\
\frac{1}{2} a, 1+a-b, 1+a-c, 1+a-d, b+c+d-a-n, a+n+1
\end{array} ; s t\right] d t \\
= & \frac{\Gamma(a)(1+a)_{n}(a-b-c+1)_{n}(a-b-d+1)_{n}(a-c-d+1)_{n}}{s^{a}(1+a-b)_{n}(1+a-c)_{n}(1+a-d)_{n}(1+a-b-c-d)_{n}}, \\
& \int_{0}^{\infty} e^{-s t} t^{a-1} \\
= & \frac{(b-a-1)(c-a-1)(d-a-1)(n+2+a-b-c-d)(a+n) \Gamma(a)}{n F_{7}\left[\begin{array}{c}
b, \\
\frac{1}{2}(a+3), c, d, 2 a-b-c-d+n+3,1-n, \\
\frac{1}{2}(a+1), a-b, 1+a-c, 1+a-d, b+c+d-a-n-1, a+n+1,2
\end{array} ; s t\right] d t} \\
\times & \left\{\begin{array}{c}
(a)_{n}(a-b-c+2)(1-c)(1-d)(b+c+d-2 a-2-n) \\
(1+a-b)_{n}(1+a-b-d)_{n}(1+a-d)_{n}(3+a-b-c-d)_{n}
\end{array}\right\}
\end{aligned}
$$

and

$$
\begin{aligned}
& \int_{0}^{\infty} e^{-s t} t^{a-1} \\
\times & { }_{7} F_{7}\left[\begin{array}{c}
b, \frac{1}{2}(a+4), c, \quad d, 2 a-b-c-d+n+5,2-n, 1 \\
\frac{1}{2}(a+2), 3+a-b, 3+a-c, 3+a-d, b+c+d-a-n-2, a+n+1,3
\end{array} ; s t\right] d t \\
= & \frac{(a-2)(b-a-2)_{2}(c-a-2)_{2}(d-a-2)_{2}}{s^{a}(a+2)(1-a)_{2}(1-b)_{2}(1-c)_{2}(1-d)_{2}} \\
\times & \frac{(-a-n)_{n}(3+n+a-b-c-d)_{2} \Gamma(a)}{(n-1)_{2}(b+c+d-2 a-4-n)_{2}} \\
\times & \left\{\frac{(a-1)_{n}(a-b-c+3)_{n}(a-b-d+3)_{n}(a-c-d+3)_{n}}{(a-b+1)_{n}(a-c+1)_{n}(a-d+1)_{n}(a-b-c-d+5)_{n}}\right. \\
& \left.+\frac{n a(b-2)(c-2)(d-2)(2 a-b-c+n+3)}{(a-b+1)(a-c+1)(a-d+1)(b+c+d-a-n-4)(n+a-1)}-1\right\} .
\end{aligned}
$$

Similarly, other results can be obtained from Theorems 32-35.

Remark 6. For evaluation of Eulerian's type integrals involving generalized hypergeometric functions by employing the summation theorems, Equations (16)-(26), we refer an interesting paper by Jun et al. [25].

\section{Concluding Remark}

In the theory of generalized hypergeometric functions, classical summation theorems such as those of Gauss, Gauss second, Kummer, Bailey, Dixon, Watson, Whipple, Saalschütz and Dougall play a key role. Applications of the above-mentioned classical summation theorems are well-known. Very recently, Masjed-Jamei and Koepf established interesting and useful generalizations of the above-mentioned classical summation theorems in the most general form.

In this paper, an attempt has been made for providing a list of several Laplace-type integrals involving generalized hypergeometric functions ${ }_{p} F_{p}$ for $p=2,3,4,5$ and 7 in the most general forms which would serve as key formulas from which, on specializing the parameters, lead to several results, some of which are known and others are believed to be new. The results established in this paper are simple, interesting, easily proven and may be potentially useful. 
We conclude this section by remarking that other applications of the generalized summation theorems due to Masjed-Jamei and Koepf are under investigations and the same will form a part of the subsequent paper in this direction.

Author Contributions: All authors contributed equally to writing of this paper. All authors read and approved the final manuscript.

Funding: The research work of Insuk Kim is supported by Wonkwang University research fund in 2019.

Acknowledgments: The authors are grateful to the referees for making certain very useful suggestions which led to a better presentation of the paper.

Conflicts of Interest: The authors declare that they have no competing interests.

\section{References}

1. Andrews, G.E.; Askey, R.; Roy, R. Special Functions. In Encyclopedia of Mathematics and Its Applications; Cambridge University Press: Cambridge, UK, 1999; Volume 71.

2. Bailey, W.N. Generalized Hypergeometric Series; Cambridge University Press: Cambridge, UK, 1935; Reprinted by Stechert-Hafner, New York, NY, USA, 1964.

3. Oberhettinger, F.; Badi, L. Tables of Laplace Transforms; Springer: Berlin, Germany, 1973.

4. Rainville, E.D. Special Functions; The Macmillan Company: New York, NY, USA, 1960; Reprinted by Chelsea Publishing Company, Bronx, NY, USA, 1971.

5. Koepf, W. Hypergeometric Summation: An Algorithmic Approach to Summation and Special Function Identities, 2nd ed.; Springer: London, UK, 2014.

6. Bromwich, T.J. An Introduction to the Theory of Infinite Series; Macmillan: New York, NY, USA, 1948.

7. Knopp, K. Theory and Applications of Infinite Series: Hafner: New York, NY, USA, 1949; Reprinted by Dover, 1990.

8. Luke, Y.L. The Special Functions and Their Approximations; Academic Press: New York, NY, USA, 1969; Volume 1.

9. Bailey, W.N. Products of Generalized Hypergeometric Series. Proc. Lond. Math. Soc. 1928, 28, $242-254$. [CrossRef]

10. Prudnikov, A.P.; Brychkov, Yu.A.; Marichev, O.I. More Special Functions; Integrals and Series; Gordon and Breach Science Publishers: Amsterdam, The Netherlands, 1990; Volume 3.

11. Masjed-Jamei, M.; Koepf, W. Some Summation Theorems for Generalized Hypergeometric Functions. Axioms 2018, 7, 38. [CrossRef]

12. Kim, Y.S.; Rakha, M.A.; Rathie, A.K. Extensions of Certain Classical Summation Theorems for the Series ${ }_{2} F_{1},{ }_{3} F_{2}$ and ${ }_{4} F_{3}$ with Applications in Ramanujan's Summations. Int. J. Math. Math. Sci. 2010, 2010, 309503. [CrossRef]

13. Lavoie, J.L.; Grondin, F.; Rathie, A.K. Generalizations of Watson's theorem on the sum of $\mathrm{a}_{3} F_{2}$. Indian J. Math. 1992, 34, 23-32.

14. Lavoie, J.L.; Grondin, F.; Rathie, A.K.; Arora, K. Generalizations of Dixon's Theorem on the sum of $\mathrm{a}_{3} F_{2}(1)$. Math. Comp. 1994, 62, 267-276.

15. Lavoie, J.L.; Grondin, F.; Rathie, A.K. Generalizations of Whipple's theorem on the sum of a ${ }_{3} F_{2}$. J. Comput. Appl. Math. 1996, 72, 293-300. [CrossRef]

16. Rakha, M.A.; Rathie, A.K. Generalizations of classical summation theorems for the series ${ }_{2} F_{1}$ and ${ }_{3} F_{2}$ with applications. Integral Transform. Spec. Funct. 2011, 22, 823-840. [CrossRef]

17. Davis, B. Integral Transforms and Their Applications, 3rd ed.; Springer: New York, NY, USA, 2002.

18. Doetsch, G. Introduction to the Theory and Applications of the Laplace Transformation; Springer: New York, NY, USA, 1974.

19. Erdelyi, A.; Magnus, W.; Oberhettinger, F.; Tricomi, F.G. Tables of Integral Transforms; McGraw Hill: New York, NY, USA, 1954; Volume I-II.

20. Slater, L.J. Confluent Hypergeometric Functions; Cambridge University Press: Cambridge, UK, 1960.

21. Deepthi, P.; Prajapati, J.C.; Rathie, A.K. New Laplace transforms of the ${ }_{2} F_{2}$ hypergeometric function. J. Fract. Calc. Appl. 2017, 8, 150-155.

22. Parmar, R.K.; Purohit, S.D. Certain integral transforms and fractional integral formulas for the extended hypergeometric functions. TWMS J. Appl. Eng. Math. 2017, 7, 74-81. 
23. Kim, Y.S.; Rathie, A.K.; Civijovic, D. New Laplace transforms of Kummer's confluent hypergeometric functions. Math. Comput. Model. 2012, 55, 1068-1071. [CrossRef]

24. Kim, Y.S.; Rathie, A.K.; Lee, C.H. New Laplace transforms for the generalized hypergeometric functions ${ }_{2} F_{2}$. Honam Math. J. 2015, 37, 245-252. [CrossRef]

25. Jun, S.; Kim, I.; Rathie, A.K. On a new class of Eulerian's type integrals involving generalized hypergeometric functions. Aust. J. Math. Anal. Appl. 2019, 16, 10.

(C) 2019 by the authors. Licensee MDPI, Basel, Switzerland. This article is an open access article distributed under the terms and conditions of the Creative Commons Attribution (CC BY) license (http://creativecommons.org/licenses/by/4.0/). 